\title{
Microbiome: A Supportive or a Leading Actor in Lung Cancer?
}

\author{
Diana Martins ${ }^{a-f}$ Fernando Mendes ${ }^{c-f}$ Fernando Schmitt ${ }^{a, b}, g$ \\ aI3S, Instituto de Investigação e Inovação em Saúde, University of Porto, Porto, Portugal; ${ }^{b}$ IPATIMUP, Institute of \\ Molecular Pathology and Immunology of University of Porto, Porto, Portugal; ' Polytechnic Institute of Coimbra, \\ ESTESC-Coimbra Health School, Department of Biomedical Laboratory Sciences, Coimbra, Portugal; ${ }^{\text {dUniversity }}$ \\ of Coimbra, Coimbra Institute for Clinical and Biomedical Research (iCBR) area of Environment Genetics and \\ Oncobiology (CIMAGO), Biophysics Institute of Faculty of Medicine, Coimbra, Portugal; ' University of Coimbra, \\ Center for Innovative Biomedicine and Biotechnology (CIBB), Coimbra, Portugal; ${ }^{f}$ Clinical Academic Center of \\ Coimbra (CACC), Coimbra, Portugal; ${ }^{9}$ FMUP, Faculty of Medicine, University of Porto, Porto, Portugal
}

\section{Keywords}

Microbiome · Lung · Lung cancer · Dysbiosis · Treatment

\begin{abstract}
Lung cancer is still the leading cause of cancer death worldwide. Despite the major diagnostic and therapeutic innovations, the effect on mortality has been modest and the overall survival is still poor. Better understanding of the pathology of these tumors is necessary in order to develop personalized therapeutic strategies in lung cancer patients. Human microbiome has been associated with normal physiology and function, and increasing evidence points towards a key role of the microbiome in promoting the progression of lung disease. Studies have shown that although poorly understood, lung has a distinctive microbiome that may an important role in lung cancer development and progression, and interactions between microbial populations have the potential to influence disease, suggesting that microbiome can be an emerging target in cancer therapeutics. We will review mechanisms how the lung microbiota influences carcinogenesis, focusing on the bacterial dysbiosis and inflammation. Moreover, we will discuss the link between the microbiome and cancer and the consequences induced by the
\end{abstract}

immune system, as the host microbiota plays an essential role in activating and modulating the immune response. We summarize current research advances in the lung microbiome and demonstrate the potential to exploit microbiome as a mechanism to prevent carcinogenesis and modulate therapeutic strategy, suggesting microbiome as a valuable approach in lung cancer patients.

(c) 2020 S. Karger AG, Basel

\section{Introduction}

Lung cancer still is the leading cause of cancer incidence and mortality worldwide. In both sexes combined, lung cancer is the most commonly diagnosed form of cancer (11.6\% of the total cases) [1]. Lung cancer is exceedingly heterogeneous and comprises several subtypes with pathological and clinical relevance. Among the reasons for its high mortality is the fact that about $70 \%$ of lung cancer patients are diagnosed in advanced-stage disease (stage III or IV) [2]. Based on the above, detection, diagnosis, and subsequent treatment of lung cancer are critical to patient outcomes and survival [2-4]. Recently, the development of molecularly targeted therapies and

$\begin{aligned} & \text { karger@karger.com } \\ & \text { www.karger.com/pat }\end{aligned}$
Karger $\%$

Fernando Schmitt 
treatment selection based on molecular alterations (epidermal growth factor receptor, programmed cell death protein 1, and anaplastic lymphoma kinase) revealed powerful tools to be used in lung cancer treatment. This allows tailored personalized therapies to subsets of specific genomic abnormalities across different tumor types and targeting tumor microenvironment components, namely the immune system and the antitumor immunity $[3,5]$. Novel directions include immunotherapy, which has opened the door for new opportunities for lung cancer therapy. Although mortality rates have decreased over the last years due to advances in detection and treatment, lung cancer remains a challenge.

The microbiome of the humans is the commensal, symbiotic, and pathogenic microorganisms (protozoa, fungi, bacteria, and viruses) that share our body space and form organ-specific microbial communities. The microbiome composition and size can vary from body parts, being affected by the host and environmental factors [6]. Microbiome also includes the means of accessing the organisms DNA, its metabolites, RNA species and proteins, supporting the central role of microbiome in human health and disease [7].

The human microbiome plays a crucial role in physiology, with some of the microbiome organisms considered beneficial [8]. However, some components of the microbiome are considered especially detrimental to human health [8]. Several studies demonstrated that bacteria and viruses can be associated with chronic inflammation leading to an increased risk of lung and colon cancer [6, 9-12]. Mycobacterium tuberculosis has been associated with lung cancer, Bacteroides fragilis and Fusobacterium nucleatum have been associated with colon cancer. Also, Helicobacter pylori in a gastric cancer model and human papillomavirus and Chlamydia trachomatis in cervical cancer $[5,12]$ are examples of the importance of microbiome environment in cancer development.

Involvement of lung microbiome has been largely ignored and it was a common belief that healthy lungs were sterile, perhaps because the access to the lower airways was difficult without invasive procedures [10]. One of the first studies in lung microbiome demonstrated that the lower airways presented a bacterial density similar to the upper small bowel, suggesting that the results reflected contamination from the bronchoscope passage through the upper respiratory tract [13]. Recent studies have shown that in fact, there is a rich and diverse microbiome in healthylungs, together with the recognition that its alterations influence lung disease, suggesting an underlying event of microbiome in lung disease development and progression $[5,14]$.

\section{Lung Microbiome}

The existence of a healthy lung microbiome is now generally accepted. The human airways harbor bacteria and other microbes in rich surroundings [15] that are distinct in composition from the microbiota seen at other organs. Innovation in the techniques of culture-independent microbial identification have shown that the lungs contain diverse and dynamic communities of microbes [16]. 16S rRNA gene sequencing became the first molecular tool to examine the bacterial communities present within the human body [17] and is widely used in environmental studies for the whole microbial population [18]. Shotgun sequencing is also used and analyzes the composition and allows metagenomics of microbial community members at high resolution [19]. The healthy lung microbiome is the result of the migration of microorganisms from the upper airways or the local growth conditions [20] and according to 16S rRNA studies, the healthy lung microbiome is characterized by the presence of Firmicutes, Bacteroidetes, Proteobacteria, Fusobacteria, and Actinobacteria $[16,21,22]$. The oropharynx and intrathoracic airways mimic the microbiome observed in lung [13]; however, nasal swabs do not reflect the lower airway microbiome [23]. Surprisingly, the nasal microbiota seems to resemble the skin more and contributes little to lung communities [24]. In disease conditions, the microbiome associated to oropharynx does not match to the lower airways and differences can be observed also within the lung microbiome [13].

Considering the reduced nutrient status, the amount of bacteria present in healthy lungs is surprisingly low but can increase in response to respiratory disease [25], reflecting important changes not only in $\mathrm{pH}$, oxygen tension, and temperature but also in immune defense responses and inflammation [26]. The microbiological determinants of the lung microbiota change during acute and chronic lung disease $[13,27]$ and the relationship between microorganisms and inflammatory lung diseases is already well established [10]. One of the best examples is tuberculosis. The high incidence of this disease is associated with treatment resistance provided by the composition of Mycobacterium tuberculosis structure [10]. Mycosides prevent the bacteria from elimination by human macrophages, and even after phagocytosis, the viral function is maintained in the macrophage's endosomes [28]. These mechanisms induce chronical inflammation of the lung tissue that can lead to fibrosis and lung cancer [29]. Besides Mycobacterium tuberculosis, Haemophilus influenzae, Moraxella catarrhalis, Streptococcus pneumoniae, 


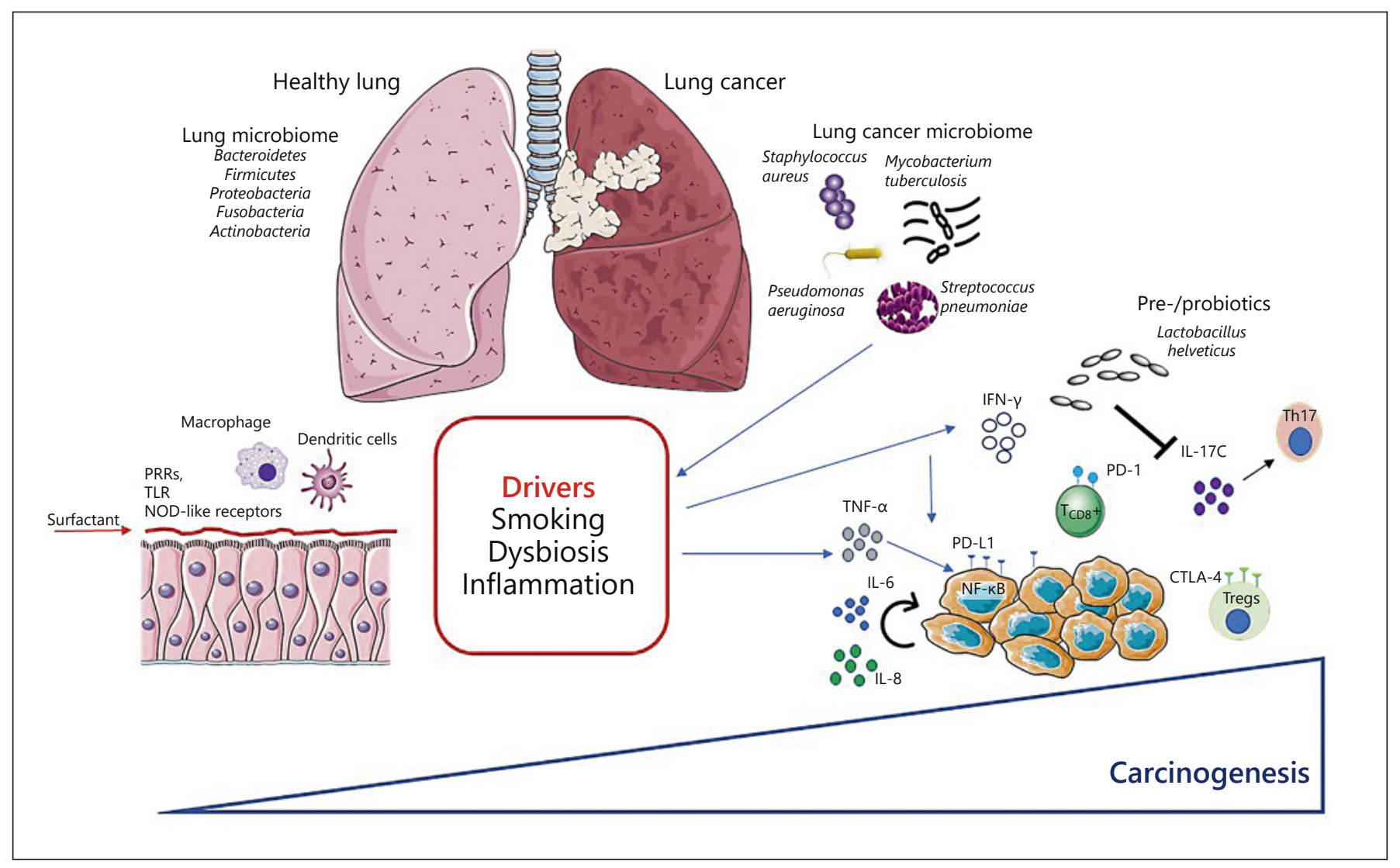

Fig. 1. Interplay between lung microbiome and cancer: carcinogenesis, drivers, and treatment in lung carcinoma. CTLA-4, cytotoxic T lymphocyte-associated protein 4; IFN- $\gamma$, interferon gamma; IL, interleukin; NF- $\kappa$ B, nuclear factor-kappa light-chain enhancer of activated B cells; NOD-like receptors, nucleotide binding oligomerization domain-like receptors; PD-1, programmed death 1; PD-L1, programmed death ligand 1; PRRs, pattern recognition receptors; Th17, T helper cells; TLR, toll-like receptor; TNF- $\alpha$, tumor necrosis factor- $\alpha$; Tregs, regulatory T cells.

Haemophilus parainfluenzae, Staphylococcus aureus, and Pseudomonas aeruginosa are also described as associated with chronic lung inflammation, as shown in Figure 1. The persistence of these agents activates the human immune system; the microbial community of the lungs becomes unstable, promoting cell lysis products dispersion, such as lipopolysaccharides (LPSs) that can affect the immune system and activate the pro-inflammatory cascade [10]. Moreover, linked to this inflammatory process is a substantial increase in the production of mucus, representing an increased microbial source of nutrients and enabling the growth of opportunistic microbial organisms [25]. In asthma, colonization with Streptococcus was a strong predictor of the disease [30]. In fact, the microbiome of these asthma patients suggests the presence of Haemophilus and Neisseria spp., accompanied by reductions in commensals, such as Prevotella and Veillonella spp. [31]. In chronic obstructive pulmonary disease
(COPD), although the microbiome does not to change with advanced stages of disease, Streptococcus, Pseudomonas, and Haemophilus are present in lung microbiome. In cystic fibrosis, the extensive production of mucus and the inflammatory machinery enable the environment to the colonization of Staphylococcus aureus, Haemophilus influenzae, and Pseudomonas aeruginosa [32]. Overall, it appears that the lung is constantly exposed to the environment and microbiome evolves in consequence of different environments and alterations in lung microbiome associated with disease stages of development.

\section{Lung Cancer Microbiome}

The microbiome has been implicated in cancer in a variety of specific ways, and the microbial factors that may be responsible for lung cancer development are still 
not well known. The hypothesis that microbiome can be directly oncogenic, through promotion of oncogenic mucosal inflammation or promoting metabolic/immune dysregulation or even modulating cancer immunity/therapy, is important to unravel the role of microbiome in lung cancer [33]. Pathogenic microorganisms are indeed able to drive inflammation in a lung cancer model [10], such as Haemophilus influenzae, Enterobacter spp., E. coli, Pneumococcus, and Legionella [10, 34]. Airway brushings of lung cancer patients demonstrate a decrease in a-diversity and increase of Streptococcus spp. compared with brushings from the contralateral non-cancerous lung [35]. Tumoral and non-tumoral brushings showed lower diversity and altered compositions, suggesting a significant variability in the airway microbiome in lung cancer patients [33]. Sputum samples from lung cancer patients presented more Granulicatella, Abiotrophia, and Streptococcus [36] and analysis of bronchoalveolar fluid from patients with lung cancer suggested that at the levels of the phyla, Firmicutes and TM7 were increased in patients with lung cancer [5]. An enrichment of Streptococcus and Veillonella in lung cancer patients was associated with activation of phosphoinositide 3-kinases (PI3K) and extracellular signal-regulated kinases (ERK) signaling pathways, suggesting the role of the microorganisms in proliferation, migration, and invasion [37]. In addition, Haemophilus influenza may promote tumor formation mediated by IL-17C and neutrophil infiltration and can increase metastatic growth of lung cancers in combination with cigarette smoke [38].

An important association between the lung microbiome and histologic classification of lung cancer was also observed. Analyzing 165 cases of lung cancer, the genus Thermus was more prevalent in adenosarcomas than in squamous cell carcinoma [39]. Moreover, Acidovorax, Klebsiella, Rhodoferax, Comamonas, and Polarmonas are more frequently associated with small-cell carcinoma and are not observed in adenocarcinoma cases. Pseudomonas shows a correlation with adenocarcinoma [10]. Microbiome has also been correlated with distant lung metastasis in lung cancer. In fact, genus Thermus, mainly Streptococcus, was more prevalent in stage IV lung cancer compared to stages I-III and with increased hepatic metastasis from non-small cell lung cancer (NSCLC) [40].

Interestingly, the bacteria found in bronchial fluid from lung cancer patients can originate in the mouth, suggesting an important connection between the oral microbiome and lung cancer [41]. In fact, the salivary microbiome was different from the saliva of lung cancer patients, particularly in microorganisms such as Capnocy-

Microbiome and Lung Cancer tophaga, Selenomonas, Veillonella, and Neisseria [42]. Molecular studies indicate that Capnocytophaga and Veillonella were more frequent in lung cancer patients, suggesting that these organisms can potentially be considered lung cancer biomarkers [5].

Despite the few studies in lung cancer microbiome, alterations in Granulicatella, Streptotococcus, and Veillonella seem to be common events, suggesting that microbial community changes reflect biochemical alterations in lung cancer linked to an increase in anaerobic environment [9].

Recently, an association between the gut and lung microbiota has been discovered [13]. This interesting concept of gut-lung axis suggests that immune cells, such as $\mathrm{T}$ and $\mathrm{B}$ cells, activated through inflammation mechanisms due to gut microbiota, cross the gastrointestinal tract, spread to the lungs by lymphatic or hematogenous paths, activate lung immune cells, and induce several respiratory diseases [43]. Moreover, chronic lung diseases such as cancer are associated with a dysbiotic airway microbiota and commonly occur alongside gastrointestinal disorders $[44,45]$ and individuals with irritable bowel syndrome sometimes have impaired lung function [14], suggesting that changes in one compartment could affect the other compartment regarding microbial composition or function. The previous model suggests that microbiome can reprogram the immune microenvironment of a specific organ. Bacteria can spread from a necrotic, tumor environment from adjacent, distant mucosal cavities or body fluids and may alter the lung niche and decrease immune surveillance [33].

Moreover, the metabolites produced by the bacteria and the host organism can modify the metabolism and inflammation [33]. For example, butyrate, a fermentation product of fiber, is indispensable to health colonic function and it is associated with anti-inflammatory effects and is able to decrease tumorigenesis [46]. Butyrate also increases mucin production and increases intestinal barrier function through claudin-1 tight junction and therefore reduction in butyrate levels is associated to dysbiotic changes and increases intestinal permeability to bacteria [47].

\section{Drivers of Lung Cancer Microbiome}

Unlike the genetic drivers and mutations that are associated with different types of lung cancer [48], the microbial factors that may be responsible for lung cancer development are not well understood. However, there are 
key player mechanisms for the tumorigenic potential of the lung microbiome [6], primarily, the establishment of an inflammatory niche that promotes carcinogenesis, with a multifactorial etiopathogenesis, including environmental factors and chronic diseases [6]. For example, COPD creates an opening for microbial dysbiosis (a state of microbial imbalance) and colonization, sustaining a chronic inflammatory milieu, often leading to the cancer. Inflammation of the lung has direct effects on epithelial cells and is associated with a loss of epithelial integrity, resulting in the leaking of serum proteins into the airways. As a result, bacterial products and formyl peptides function as powerful chemoattractants for neutrophils and monocytes that migrate from alveoli [6] and contribute to chronic inflammation, parenchymal lung damage, and loss of alveolar attachments [49]. In vitro studies suggest that in an inflammation context, elastase, cathepsin $\mathrm{G}$, and proteinase 3 affect the integrity of the epithelial layer, increasing mucus secretion and inducing the synthesis of chemoattractant chemokine IL- 8 from respiratory epithelial cells [50]. Additionally, IL-6 and IL-8 are involved in tumorigenesis acting directly on lung epithelial cells activating the NF- $\mathrm{BB}-1$ pathway [51] and stimulating cancer cell proliferation, migration, and invasion [50], as observed in Figure 1. In lepidic growth adenocarcinoma, the presence of an increased number of neutrophils in BAL fluid was correlated with IL-8 level and associated with a poor outcome [50]. In addition, an interesting study suggests that $H$. pylori is also associated with lung cancer development, inducing the production of proinflammatory factors including IL-1, IL-6, and tumor necrosis factor. This inflammation may develop into chronic bronchitis that frequently accompanies lung cancer [52], as shown in Figure 1.

Microbiome diversity increases with tobacco smoking [53]. Smoking is one of the most important risk factors associated with lung cancer, due to the formation of DNA adducts that promote mutations [9] or through the induction of chronic inflammation [54]. Microbiome can be related since these mechanisms induce the production of reactive oxygen species, nitric oxide, interleukins, and interferons, mediated by leukocytes, promoting genomic instability and carcinogenesis [55].

The link between commensal microbiota and the host immune system is clear and can act as regulator, shaping the adaptive immunity $[33,56]$. Recently, new evidence suggested that dysbiosis of the microbiota may also play a vital role in carcinogenesis at multiple levels affecting metabolic, inflammatory, or even immune responses. The homeostasis of the lung microbiome occurs with bal- ance between immune sensing and tolerance of the microbiome [56, 57]. The immune system of the lung can defend itself against pathogen invasion, using three major pathways. The first is the increase of alveolar surfactant. The second is mediated by an epithelial cell layer that blocks pathogen translocation with the contribution of pattern recognition receptors, including toll-like receptor (TLR) and nucleotide binding oligomerization domainlike receptors, which can be found on epithelial cells, macrophages, and dendritic cells, preventing the overload of metabolites or pathogens $[58,59]$. The activation of downstream inflammatory signal pathways is activated to eliminate pathogens. As an example, the relationship between Mycobacterium tuberculosis and lung cancer was mechanically associated through the chronic inflammation-associated carcinogenesis according to previous studies $[56,60]$. This might be due to persistent infection by tuberculosis, which can induce the production of tumor necrosis factor- $\alpha$ and lead to pulmonary inflammation. Additionally, pulmonary fibrosis caused by tuberculosis led to synthesize extracellular matrix, which is involved in the development of lung cancer. Another good example is how the enterotoxigenic Bacteroides fragilis triggers the activation of STAT3 by a selective subtype of $\mathrm{T}$ cells, the $\mathrm{T}$ helper type 17 (Th17) response in mice, demonstrating that human commensal bacteria can induce carcinogenesis via a Th17-dependent pathway [56, 60,61 . Moreover, microbiota induced Th17 cells to support lung cancer cell proliferation and angiogenesis [56, $62,63]$. The activation of the tumor-associated microbiota and TLR signaling stimulates the calcineurin expression and nuclear factor of activated $\mathrm{T}$ cells, which endures the survival and proliferation of cancer stem cells [56]. Arpaiai et al. [64] and García-Castillo et al. [62] demonstrated that microbial-derived butyrate could increase regulator $\mathrm{T}$ cells by activating the forkhead box P3 and $\mathrm{G}$ protein coupled receptors, as shown in Figure 1.

Several bacterial molecules (bacteriotoxins) have been associated with tumor development and genotoxicity [6]. For example, cytolethal distending toxin, cytotoxic necrotizing factor 1, and Bacteroides fragilis have been associated with the disruption of DNA repair system [65]. Chemicals produced by bacteria such as superoxide dismutase are also associated with genomic instability [66]. An in silico study demonstrated that a cyanobacteria toxin was able to increase PARP1 enzyme and decrease CD36 protein level in a mouse model of NSCLC [67]. TLR4 stimulation with inactivated $E$. coli promotes migration and metastases on NSCLC cells in vivo, mediated by p38 MAPK and ERK1/2 signaling [68], suggesting that bacte- 
rial released products can have an important effect in carcinogenesis.

To summarize, the appearance of dysbiosis or malignancy is likely the product of a dynamic interaction between various immune, microbial, and environmental factors. At least one of these acts as an initiator and the other factors follow the trigger. Therefore, it remains difficult to reach any conclusions regarding the true etiology of disease, what might be the best intervention, and, more importantly, the prevention approach.

\section{Microbiome as a Therapeutic Target in Lung Cancer}

Given the role of microbiome in cancer development and progression, the modulation of microbiome can be used as a therapeutic strategy in lung cancer treatment. The concept of administration of microbial compounds to improve human health is not new, but the administration of microbes could in fact have beneficial effects [69]. In order to modulate the microbiome, the use of pre-/ probiotics has been suggested to synergize the effects of bacteria and substrates, promoting a health benefit and also supporting a healthy digestive tract [70-73]. Manipulation of microbiota may also be useful in cancer prevention. A recent study demonstrated that the intake of a probiotic supplement containing Lactobacillus helveticus could decrease IL-17, reduce T cells, and suppress tumor formation, maybe due to gut microbiome alteration [70], as observed in Figure 1.

Alterations in the microbiome can also affect cancer growth, as demonstrated in a study where probiotic supplementation decreased tumor cell proliferation and downregulated nuclear factor-kappa light-chain enhancer of activated B cells activation in a mouse model of inflammation-induced colon tumors [70]. Another study in a murine melanoma model demonstrated that probiotic treatment could promote immunity against lung metastasis, suggesting also a clue of microbiome relevance in cancer recurrence [74]. Microbiome can also be used in addition to standard cancer regimens [33]. In a mouse lung cancer study, antibiotics decreased the effectiveness of cisplatin-based regimen, whereas Lactobacillus decreased tumor size and improved survival [75]. Taken together, these studies suggest that microbiome may be useful in lung cancer treatment as a preventive enhancer of cancer-related and therapy-related effects.

There is growing evidence supporting that microbiome can affect immunotherapy strategies. In patients with advanced NSCLC, antibiotic therapy decreased the effectiveness of the treatment, suggesting that patients who recently received antibiotics had a higher risk of progressive disease and shorter PFS and OS [33]. Also, the use of antibiotics such as penicillin and cephalosporins is demonstrated to increase the risk of lung, gastric, breast, and prostate cancer, regardless of smoking status and number of infections, proposing that microbiome alterations may have an important role in cancer development [76]. The question that remains to be answered regarding the reduction of immune checkpoint inhibitor (ICI) treatment in lung cancer patients is whether it is an effect of gut-to-lung axis microbiome or a direct anti-neoplastic effect [76].

ICIs target programmed death 1 (PD-1), programmed death-ligand 1 (PD-L1), and cytotoxic T lymphocyte-associated protein 4 (CTLA-4), examples of monoclonal antibodies used in cancer treatment, especially in advanced NSCLC [53, 77-79]. Anti-tumor therapy can use immune system to control and eliminate cancer cells, namely through specific CD8+ cytotoxic $\mathrm{T}$ cells with capacity to identify and recognize specific tumor antigens, inducing cell death [53]. The administration of ICIs releases a $\mathrm{T}$ cell-mediated immune response, mainly due to the inhibition of the interaction of $\mathrm{T}$ cell-suppressing receptors with their corresponding ligand present on tumor cells [80]. On the other hand it is known that PD-L1 expressing cancer cells induces caspase-dependent cell death of co-cultured activated CD8+ cytotoxic T cells and an anti-human PD-L1 monoclonal antibody can block this interaction [80], as shown in Figure 1.

Recent studies demonstrated that microbiome may be a leading actor regarding response to cancer immunotherapy [80-84]. The importance of microbiome linked to ICIs was observed in mouse models, where gut microbiome influences the response to cancer immunotherapy (targeting PD-1 and CTLA-4) [80]. Remarkably, the oral administration of Bacteroides fragilis together with Burkholderia cepacia or Bacteroides thetaiotaomicron stimulated anti-CTLA-4 immunotherapy [80]. Moreover the oral probiotic supplementation with Bifidobacterium seems to re-sensitize tumors to anti-PD-L1 therapy in mice, inducing dendritic cell maturation with anti-tumor CD8+ cytotoxic T cell activity [80], increasing response to ICI treatment.

The gut-lung axis seems to play an important role in the efficacy of PD-1-based immunotherapy [79]. The early use of antibiotics has been associated with worse outcomes with ICIs, perhaps due to the intestinal barrier modification, alteration of fecal microbiota, and alteration in anti-tumor immune response [79]. Dysbiosis 
caused by antibiotic can induce resistance to ICIs with the involvement of intestinal microbiome, by reducing microbiota diversity [53]. Wojas-Krawczyk et al. [53] demonstrated that a gut microbiome with high diversity promotes Th1 lymphocyte and M1 macrophage differentiation, activation of helper and cytotoxic $\mathrm{T}$ cell as well as upregulation expression of PD-1 on lymphocytes. So antibiotics-related dysbiosis modulation and gut microbiota composition may be an important approach to improve clinical outcomes with ICIs [53]. The use of antibiotics represented a predictor of resistance to PD-1 or PD-L1 blockade, independent from classical prognostic markers in NSCLC [40]. Botticelli et al. [85] studied gut microbiome in NSCLC patients treated with nivolumab and compared them with healthy people. Using NGS method demonstrated low levels of Rikenellaceae, Prevotella, Streptococcus, Lactobacillus, Bacteroides plebeius, Oscillospira, and Enterobacteriaceae in healthy controls, while NSCLC patients responsive to therapy with nivolumab revealed increased levels of Akkermansia muciniphila, Bifidobacterium longum, Faecalibacterium prausnitzi, Propionibacterium acnes, Veillonella parvula, Staphylococcus aureus, and Peptostreptococcus and lower levels of Ruminococcus bromii, Dialister, and Sutterella. This study showed the important link between microbiome and ICIs, revealing that microbiome affects the immunotherapy response [53]. Therefore, the high diversity of the microbiome positively influences the ICI efficacy, restoring microbiome composition in cancer patients after antibiotics therapy consequently with dysbiosis, potentiating immunotherapy.

\section{Challenges in Lung Cancer Microbiome}

Lung remains a clinical challenge in diagnosis, treatment, and in microbiome assessment. Microbiome is influenced by genetic, environmental, and dietary factors [33]. For example, there are differences in microbial composition associated with gender [86, 87] and smoking habits. Smoking has an established association with lung cancer [12] and it is an intrinsic component of lung microbiome [40], in addition to the gut microbiome [88]. Tobacco and professional exposure inhaling requires cellular remodeling and adaptation of the basal cells of the lungs [7], suggesting that a specific microbial environment could enhance the neoplastic process. The lung microbiome also depends on the region of the lung sampled [89], suggesting the variability within the lung, but also differences in microenvironment, such as $\mathrm{pH}$ levels, oxy- gen tension, and immune conditions, are indeed factors that promote microorganism colonization in the lung $[90,91]$. Moreover, global environmental and geographical alterations play vital roles in the gut microbiota; however, these effects seems controversial in lung microbiome [40].

Genetic heterogeneity has been associated with lung cancer [92]. Tumor heterogeneity has been explained by genetic events (intra- and inter-tumor) and also by nongenetic events driven by external or internal forces allowing the growth of cell subpopulations related to microenvironment and interactions with immune and stromal cells or with matrix components [93]. Tumor heterogeneity has an important impact on tumor classification and also on defining prognosis and therapy decision in lung cancer patients. Previously, it was demonstrated that microbiome is associated with specific histological subtypes of lung cancer. Based on this fact, it is reasonable to speculate that microbiome can also play a role in tumor heterogeneity.

Another important challenge in lung cancer microbiome is contamination. It can occur in the sampling procedure, at DNA extraction, and during the PCR amplification and in sequencing [94]. Samples from the lung can contain relatively few bacteria [95] but contamination from swabs or bronchoscopy is important to minimize. Sputum is clinically used in airway sample collection despite the contamination from upper airways and saliva. In lung, in order to obtain reliable results, we strongly support the idea of using controls in molecular microbiome [17].

\section{Conclusions}

In conclusion, the microbiome seems to play a complex role in human lung model and can be an emerging target in cancer development and therapeutics. However, the considerable variation in the microbiota and the genetic susceptibility of hosts lead to the heterogeneity of studies attempting to establish cause by specific bacteria. In lung cancer model, it is possible that either dysbiotic bacteria or their products cause alterations in the mucosa surfaces, including direct bacterial invasion, increase in neutrophil and other immune cell infiltrates, upregulation of cytokines, and oncogenic pathways. Alterations in the immune microenvironment may also explain why microbiome composition affects the efficacy of ICIs and why antibiotic and pre-/probiotic treatment may influence cancer therapies. Considering the extended number 
of potential connections between the microbiome and cancer, it is important to better understand the microbiome in the context of lung disease pathogenesis and treatment.

\section{Conflict of Interest Statement}

The authors have no conflicts of interest to declare.

\section{Funding Sources}

The study did not receive any funding.

\section{Author Contributions}

Fernando Schmitt conceived the manuscript. Diana Martins and Fernando Mendes wrote the manuscript with supervision by Fernando Schmitt. All authors contributed to the design, discussed the items, and contributed to the final manuscript.

\section{References}

1 Bray F, Ferlay J, Soerjomataram I, Siegel RL, Torre LA, Jemal A. Global cancer statistics 2018: GLOBOCAN estimates of incidence and mortality worldwide for 36 cancers in 185 countries. CA Cancer J Clin. 2018 Nov;68(6): 394-424.

2 Lemjabbar-Alaoui H, Hassan OU, Yang YW, Buchanan P. Lung cancer: biology and treatment options. Biochim Biophys Acta. 2015 Dec;1856(2):189-210.

3 Zugazagoitia J, Guedes C, Ponce S, Ferrer I, Molina-Pinelo S, Paz-Ares L. Current Challenges in Cancer Treatment. Clin Ther. 2016 Jul;38(7):1551-66.

4 Jacobsen MM, Silverstein SC, Quinn M, Waterston LB, Thomas CA, Benneyan JC, et al. Timeliness of access to lung cancer diagnosis and treatment: A scoping literature review. Lung Cancer. 2017 Oct;112:156-64.

5 Lee SH, Sung JY, Yong D, Chun J, Kim SY, Song JH, et al. Characterization of microbiome in bronchoalveolar lavage fluid of patients with lung cancer comparing with benign mass like lesions. Lung Cancer. 2016 Dec;102:89-95.

6 Maddi A, Sabharwal A, Violante T, Manuballa S, Genco R, Patnaik S, et al. The microbiome and lung cancer. J Thorac Dis. 2019 Jan; 11(1):280-91.

7 de Sousa VM, Carvalho L. Heterogeneity in Lung Cancer. Pathobiology. 2018;85(1-2): 96-107.

8 Chen J, Douglass J, Prasath V, Neace M, Atrchian S, Manjili MH, et al. The microbiome and breast cancer: a review. Breast Cancer Res Treat. 2019 Dec;178(3):493-6.

9 Mur LA, Huws SA, Cameron SJ, Lewis PD, Lewis KE. Lung cancer: a new frontier for microbiome research and clinical translation. Ecancermedicalscience. 2018 Sep;12:866.

10 Kovaleva OV, Romashin D, Zborovskaya IB, Davydov MM, Shogenov MS, Gratchev A. Human Lung Microbiome on the Way to Cancer. J Immunol Res. 2019 Jul;2019: 1394191.

11 Jin C, Lagoudas GK, Zhao C, Bullman S, Bhutkar A, Hu B, et al. Commensal Microbiota Promote Lung Cancer Development via $\gamma \delta$ T Cells. Cell. 2019 Feb;176(5):998-1013. e16.
12 Greathouse KL, White JR, Vargas AJ, Bliskovsky VV, Beck JA, von Muhlinen N, et al. Author Correction: interaction between the microbiome and TP53 in human lung cancer. Genome Biol. 2020 Feb;21(1):41.

13 Hilty M, Burke C, Pedro H, Cardenas P, Bush A, Bossley C, et al. Disordered microbial communities in asthmatic airways. PLoS One. 2010 Jan;5(1):e8578.

14 Clemente JC, Manasson J, Scher JU. The role of the gut microbiome in systemic inflammatory disease. BMJ. 2018 Jan;360:j5145.

15 Sze MA, Tsuruta M, Yang SW, Oh Y, Man SF, Hogg JC, et al. Changes in the bacterial microbiota in gut, blood, and lungs following acute LPS instillation into mice lungs. PLoS One. 2014 Oct;9(10):e111228.

16 Charlson ES, Bittinger K, Haas AR, Fitzgerald AS, Frank I, Yadav A, et al. Topographical continuity of bacterial populations in the healthy human respiratory tract. Am J Respir Crit Care Med. 2011 Oct;184(8):957-63.

17 Moffatt MF, Cookson WO. The lung microbiome in health and disease. Clin Med (Lond). 2017 Dec;17(6):525-9.

18 Mcmurdie PJ, Xu Z, Ursell LK, Lauber C, Song SJ, Huntley J, et al. Advancing our understanding of the human microbiome using QIIME. Vol. 531, Methods in Enzymology. 2015. 371-444 p.

19 Quince C, Walker AW, Simpson JT, Loman NJ, Segata N. Shotgun metagenomics, from sampling to analysis. Nat Biotechnol. 2017 Sep;35(9):833-44.

20 Dickson RP, Erb-Downward JR, Huffnagle GB. Towards an ecology of the lung: new conceptual models of pulmonary microbiology and pneumonia pathogenesis. Lancet Respir Med. 2014 Mar;2(3):238-46.

21 Goddard AF, Staudinger BJ, Dowd SE, JoshiDatar A, Wolcott RD, Aitken ML, et al. Direct sampling of cystic fibrosis lungs indicates that DNA-based analyses of upper-airway specimens can misrepresent lung microbiota. Proc Natl Acad Sci USA. 2012 Aug;109(34):13769-74.

22 Lemon KP, Klepac-Ceraj V, Schiffer HK, Brodie EL, Lynch SV, Kolter R. Comparative analyses of the bacterial microbiota of the human nostril and oropharynx. MBio. 2010 Jun; $1(3): 4-6$.
23 Dickson RP, Erb-Downward JR, Freeman CM, McCloskey L, Falkowski NR, Huffnagle GB, Curtis JL. Bacterial Topography of the Healthy Human Lower Respiratory Tract. mBio. 2017 Feb 14;8(1):e02287-16.

24 Dickson RP, Martinez FJ, Huffnagle GB. The role of the microbiome in exacerbations of chronic lung diseases. Lancet. 2014 Aug; 384(9944):691-702.

25 Dickson RP, Erb-Downward JR, Martinez FJ, Huffnagle GB. The Microbiome and the Respiratory Tract. Annu Rev Physiol. 2016; 78(1):481-504.

26 Schmidt A, Belaaouaj A, Bissinger R, Koller G, Malleret L, D’Orazio C, et al. Neutrophil elastase-mediated increase in airway temperature during inflammation. J Cyst Fibros. 2014 Dec;13(6):623-31.

27 Sze MA, Dimitriu PA, Hayashi S, Elliott WM, McDonough JE, Gosselink JV, et al. The Lung Tissue Microbiome in Chronic Obstructive Pulmonary Disease. Am J Respir Crit Care Med. 2012 May 15;185(10):1073-80.

28 Fogel N. Tuberculosis: a disease without boundaries. Tuberculosis (Edinb). 2015 Sep; 95(5):527-31.

29 Cukic V. The Association Between Lung Carcinoma and Tuberculosis. Med Arch (Sarajevo, Bosnia Herzegovina). 2017 Jun 1;71(3):212-4.

30 Teo SM, Mok D, Pham K, Kusel M, Serralha M, Troy N, et al. The infant nasopharyngeal microbiome impacts severity of lower respiratory infection and risk of asthma development. Cell Host Microbe. 2015 May;17(5):704-15.

31 Huang YJ, Marsland BJ, Bunyavanich S, O'Mahony L, Leung DY, Muraro A, et al. The microbiome in allergic disease: Current understanding and future opportunities-2017 PRACTALL document of the American Academy of Allergy, Asthma \& Immunology and the European Academy of Allergy and Clinical Immunology. J Allergy Clin Immunol. 2017 Apr;139(4):1099-110.

32 Mogayzel PJ Jr, Naureckas ET, Robinson KA, Mueller G, Hadjiliadis D, Hoag JB, et al.; Pulmonary Clinical Practice Guidelines Committee. Cystic fibrosis pulmonary guidelines. Chronic medications for maintenance of lung health. Am J Respir Crit Care Med. 2013 Apr; 187(7):680-9. 
33 Picardo SL, Coburn B, Hansen AR. The microbiome and cancer for clinicians. Crit Rev Oncol Hematol. 2019 Sep;141(June):1-12.

34 Laroumagne S, Salinas-Pineda A, Hermant C, Murris M, Gourraud PA, Do C, et al. Incidence et caractéristiques des colonisations des voies respiratoires lors du diagnostic de cancer bronchique: étude rétrospective de 388 cas. Rev Mal Respir. 2011 Mar;28(3):328-35.

35 Liu HX, Tao LL, Zhang J, Zhu YG, Zheng Y, Liu D, et al. Difference of lower airway microbiome in bilateral protected specimen brush between lung cancer patients with unilateral lobar masses and control subjects. Int J Cancer. 2018 Feb;142(4):769-78.

36 Hosgood HD 3rd, Sapkota AR, Rothman N, Rohan T, Hu W, Xu J, et al. The potential role of lung microbiota in lung cancer attributed to household coal burning exposures. Environ Mol Mutagen. 2014 Oct;55(8):643-51.

37 De Marco C, Laudanna C, Rinaldo N, Oliveira DM, Ravo M, Weisz A, et al. Specific gene expression signatures induced by the multiple oncogenic alterations that occur within the PTEN/PI3K/AKT pathway in lung cancer. PLoS One. 2017 Jun;12(6):e0178865.

38 Jungnickel C, Schmidt LH, Bittigkoffer L, Wolf L, Wolf A, Ritzmann F, et al. IL-17C mediates the recruitment of tumor-associated neutrophils and lung tumor growth. Oncogene. 2017 Jul;36(29):4182-90.

39 Huang D, Su X, Yuan M, Zhang S, He J, Deng Q, Qiu W, Dong H, Cai S. The characterization of lung microbiome in lung cancer patients with different clinicopathology. Am J Cancer Res. 2019 Sep 1;9(9):2047-63.

$40 \mathrm{Yu} \mathrm{G}$, Gail MH, Consonni D, Carugno M, Humphrys M, Pesatori AC, et al. Characterizing human lung tissue microbiota and its relationship to epidemiological and clinical features. Genome Biol. 2016 Jul;17(1):163.

41 Hasegawa A, Sato T, Hoshikawa Y, Ishida N, Tanda N, Kawamura Y, et al. Detection and identification of oral anaerobes in intraoperative bronchial fluids of patients with pulmonary carcinoma. Microbiol Immunol. 2014 Jul;58(7):375-81.

42 Dunn GP, Bruce AT, Sheehan KC, Shankaran V, Uppaluri R, Bui JD, et al. A critical function for type I interferons in cancer immunoediting. Nat Immunol. 2005 Jul;6(7):722-9.

43 Schuijt TJ, Lankelma JM, Scicluna BP, de Sousa e Melo F, Roelofs JJ, de Boer JD, et al. The gut microbiota plays a protective role in the host defence against pneumococcal pneumonia. Gut. 2016 Apr;65(4):575-83.

44 Wang H, Liu JS, Peng SH, Deng XY, Zhu DM, Javidiparsijani S, et al. Gut-lung crosstalk in pulmonary involvement with inflammatory bowel diseases. World J Gastroenterol. 2013 Oct; 19(40):6794-804.

45 Jess T, Horváth-Puhó E, Fallingborg J, Rasmussen HH, Jacobsen BA. Cancer risk in inflammatory bowel disease according to patient phenotype and treatment: a Danish population-based cohort study. Am J Gastroenterol. 2013 Dec;108(12):1869-76.
46 O'Keefe SJ. Diet, microorganisms and their metabolites, and colon cancer. Nat Rev Gastroenterol Hepatol. 2016 Dec;13(12):691706.

47 Wang HB, Wang PY, Wang X, Wan YL, Liu YC. Butyrate enhances intestinal epithelial barrier function via up-regulation of tight junction protein Claudin-1 transcription. Dig Dis Sci. 2012 Dec;57(12):3126-35.

48 El-Telbany A, Ma PC. Cancer genes in lung cancer: racial disparities: are there any? Genes Cancer. 2012 Jul;3(7-8):467-80.

49 Sethi S. Bacterial infection and the pathogenesis of COPD. Chest. 2000 May;117(5 Suppl 1):286S-91S.

50 Bingula R, Filaire M, Radosevic-Robin N, Bey M, Berthon JY, Bernalier-Donadille A, et al. Desired Turbulence? Gut-Lung Axis, Immunity, and Lung Cancer. J Oncol. 2017;2017: 5035371.

51 Lin WW, Karin M. A cytokine-mediated link between innate immunity, inflammation, and cancer. J Clin Invest. 2007 May;117(5):117583.

52 Koshiol J, Flores R, Lam TK, Taylor PR, Weinstein SJ, Virtamo J, et al. Helicobacter pylori seropositivity and risk of lung cancer. PLoS One. 2012;7(2):e32106.

53 Wojas-Krawczyk K, Kalinka E, Grenda A, Krawczyk P, Milanowski J. Beyond PD-L1 markers for lung cancer immunotherapy. Int J Mol Sci. 2019 Apr;20(8):E1915.

54 Balkwill FR, Mantovani A. Cancer-related inflammation: common themes and therapeutic opportunities. Semin Cancer Biol. 2012 Feb;22(1):33-40.

55 Chumduri C, Gurumurthy RK, Zietlow R, Meyer TF. Subversion of host genome integrity by bacterial pathogens. Nat Rev Mol Cell Biol. 2016 Oct;17(10):659-73.

56 Mao Q, Jiang F, Yin R, Wang J, Xia W, Dong $\mathrm{G}$, et al. Interplay between the lung microbiome and lung cancer. Cancer Lett. 2018 Feb; 415:40-8.

57 Dethlefsen L, McFall-Ngai M, Relman DA. An ecological and evolutionary perspective on human-microbe mutualism and disease. Nature. 2007 Oct;449(7164):811-8.

58 LeVine AM, Whitsett JA, Gwozdz JA, Richardson TR, Fisher JH, Burhans MS, et al. Distinct effects of surfactant protein A or D deficiency during bacterial infection on the lung. J Immunol. 2000 Oct;165(7):393440.

59 Uehara A, Fujimoto Y, Fukase K, Takada H. Various human epithelial cells express functional Toll-like receptors, NOD1 and NOD2 to produce anti-microbial peptides, but not proinflammatory cytokines. Mol Immunol. 2007 May;44(12):3100-11.

60 Liang HY, Li XL, Yu XS, Guan P, Yin ZH, He QC, et al. Facts and fiction of the relationship between preexisting tuberculosis and lung cancer risk: a systematic review. Int J Cancer. 2009 Dec;125(12):2936-44.

$61 \mathrm{Wu}$ S, Rhee KJ, Albesiano E, Rabizadeh S, Wu $\mathrm{X}$, Yen HR, et al. A human colonic commensal promotes colon tumorigenesis via activation of T helper type $17 \mathrm{~T}$ cell responses. Nat Med. 2009 Sep;15(9):1016-22.

62 García-Castillo V, Sanhueza E, McNerney E, Onate SA, García A. Microbiota dysbiosis: a new piece in the understanding of the carcinogenesis puzzle. J Med Microbiol. 2016 Dec; 65(12): 1347-62.

63 Ochoa CE, Mirabolfathinejad SG, Ruiz VA, Evans SE, Gagea M, Evans CM, et al. Interleukin 6, but not T helper 2 cytokines, promotes lung carcinogenesis. Cancer Prev Res (Phila). 2011 Jan;4(1):51-64.

64 Arpaia N, Campbell C, Fan X, Dikiy S, van der Veeken J, deRoos P, et al. Metabolites produced by commensal bacteria promote peripheral regulatory T-cell generation. Nature. 2013 Dec;504(7480):451-5.

65 Cuevas-Ramos G, Petit CR, Marcq I, Boury M, Oswald E, Nougayrède JP. Escherichia coli induces DNA damage in vivo and triggers genomic instability in mammalian cells. Proc Natl Acad Sci USA. 2010 Jun;107(25):1153742.

66 Carbonero F, Benefiel AC, Alizadeh-Ghamsari AH, Gaskins HR. Microbial pathways in colonic sulfur metabolism and links with health and disease. Front Physiol. 2012 Nov; 3(November): 448 .

67 Apopa PL, Alley L, Penney RB, Arnaoutakis $\mathrm{K}$, Steliga MA, Jeffus S, et al. PARP1 is upregulated in non-small cell lung cancer tissues in the presence of the Cyanobacterial toxin microcystin. Front Microbiol. 2018 Aug. 9(AUG): 1757.

68 Chow SC, Gowing SD, Cools-Lartigue JJ, Chen CB, Berube J, Yoon HW, et al. Gram negative bacteria increase non-small cell lung cancer metastasis via Toll-like receptor 4 activation and mitogen-activated protein kinase phosphorylation. Int J Cancer. 2015 Mar; 136(6):1341-50.

69 Helmink BA, Khan MA, Hermann A, Gopalakrishnan V, Wargo JA. The microbiome, cancer, and cancer therapy. Nat Med. 2019 Mar;25(3):377-88.

70 Markowiak P, Śliżewska K. Effects of Probiotics, Prebiotics, and Synbiotics on $\mathrm{Hu}-$ man Health. Nutrients. 2017 Sep 15;9(9): 1021.

71 Pitt JM, Vétizou M, Daillère R, Roberti MP, Yamazaki T, Routy B, et al. Resistance Mechanisms to Immune-Checkpoint Blockade in Cancer: Tumor-Intrinsic and -Extrinsic Factors. Immunity. 2016 Jun;44(6): 1255-69.

72 Jiang C, Wang H, Xia C, Dong Q, Chen E, Qiu $\mathrm{Y}$, et al. A randomized, double-blind, placebo-controlled trial of probiotics to reduce the severity of oral mucositis induced by chemoradiotherapy for patients with nasopharyngeal carcinoma. Cancer. 2019 Apr;125(7): 1081-90.

73 Redman MG, Ward EJ, Phillips RS. The efficacy and safety of probiotics in people with cancer: a systematic review. Ann Oncol. 2014 Oct;25(10):1919-29. 
74 Le Noci V, Guglielmetti S, Arioli S, Camisaschi C, Bianchi F, Sommariva M, et al. Modulation of Pulmonary Microbiota by Antibiotic or Probiotic Aerosol Therapy: A Strategy to Promote Immunosurveillance against Lung Metastases. Cell Rep. 2018 Sep;24(13):352838.

75 Gui QF, Lu HF, Zhang CX, Xu ZR, Yang YH. Well-balanced commensal microbiota contributes to anti-cancer response in a lung cancer mouse model. Genet Mol Res. 2015 May; 14(2):5642-51.

76 Derosa L, Hellmann MD, Spaziano M, Halpenny D, Fidelle M, Rizvi H, et al. Negative association of antibiotics on clinical activity of immune checkpoint inhibitors in patients with advanced renal cell and non-small-cell lung cancer. Ann Oncol. 2018 Jun;29(6): 1437-44.

77 Mooradian MJ, Gainor JF. Putting the brakes on CTLA-4 inhibition in lung cancer? Transl Lung Cancer Res. 2018 Feb;7(S1 Suppl 1): S35-8.

78 Antonia SJ, Villegas A, Daniel D, Vicente D, Murakami S, Hui R, et al.; PACIFIC Investigators. Durvalumab after Chemoradiotherapy in Stage III Non-Small-Cell Lung Cancer. N Engl J Med. 2017 Nov;377(20):1919-29.

79 Costantini A, Takam Kamga P, Dumenil C, Chinet T, Emile JF, Giroux Leprieur E. Plasma biomarkers and immune checkpoint inhibitors in non-small cell lung cancer: new tools for better patient selection? Cancers (Basel). 2019 Aug;11(9):1-14.

80 Stancu AL. Gut Microbiome and the Response to Immunotherapy in Cancer. Discoveries (Craiova). 2018 Sep;6(3):e84.
81 Rizvi NA, Brahmer JR, Ou SH, Segal NH, Khleif S, Hwu WJ, et al. Safety and clinical activity of MEDI4736, an anti-programmed cell death-ligand 1 (PD-L1) antibody, in patients with non-small cell lung cancer (NSCLC). J Clin Oncol. 2015 May;33(15 suppl):80328032.

82 Paz-Ares L, Luft A, Vicente D, Tafreshi A, Gümüş M, Mazières J, et al.; KEYNOTE-407 Investigators. Pembrolizumab plus Chemotherapy for Squamous Non-Small-Cell Lung Cancer. N Engl J Med. 2018 Nov;379(21): 2040-51.

83 Berghoff AS, Ricken G, Wilhelm D, Rajky O, Widhalm G, Dieckmann K, et al. Tumor infiltrating lymphocytes and $\mathrm{PD}-\mathrm{L} 1$ expression in brain metastases of small cell lung cancer (SCLC). J Neurooncol. 2016 Oct;130(1):1929.

84 Zhang J, Gao J, Li Y, Nie J, Dai L, Hu W, et al. Circulating PD-L1 in NSCLC patients and the correlation between the level of PD-L1 expression and the clinical characteristics. Thorac Cancer. 2015 Jul;6(4):534-8.

85 Botticelli A, Putignani L, Zizzari I, Del Chierico F, Reddel SD, Pietro F, et al. Changes of microbiome profile during nivolumab treatment in NSCLC patients. J Clin Oncol. 2018 May;36(15 suppl):e15020-15020.

86 Pala L, Nezi L, De Pas T, Pennacchioli E, Cocorocchio E, Ferrucci P, et al. Sex Differences in Efficacy and Toxicity of Systemic Cancer Treatments: role of the Microbiome. J Clin Oncol. 2019 Feb;37(5):439-439.

87 Raju SC, Lagström S, Ellonen P, de Vos WM, Eriksson JG, Weiderpass E, et al. Gender-Specific Associations Between Saliva Microbiota and Body Size. Front Microbiol. 2019 Apr; 10(APR):767.
88 Vogtmann E, Flores R, Yu G, Freedman ND, Shi J, Gail MH, et al. Association between tobacco use and the upper gastrointestinal microbiome among Chinese men. Cancer Causes Control. 2015 Apr;26(4):581-8.

89 Erb-Downward JR, Thompson DL, Han MK, Freeman CM, McCloskey L, Schmidt LA, et al. Analysis of the Lung Microbiome in the "Healthy" Smoker and in COPD. PLoS One. 2011 Feb 22;6(2):e16384.

90 Marsland BJ, Gollwitzer ES. Host-microorganism interactions in lung diseases. Nat Rey Immunol. 2014 Dec;14(12):827-35.

91 Man WH, de Steenhuijsen Piters WA, Bogaert $\mathrm{D}$. The microbiota of the respiratory tract: gatekeeper to respiratory health. Nat Rev Microbiol. 2017 May;15(5):259-70.

92 Marjanovic ND, Weinberg RA, Chaffer CL. Poised with purpose: cell plasticity enhances tumorigenicity. Cell Cycle. 2013 Sep;12(17): 2713-4.

93 Marusyk A, Polyak K. Cancer cell phenotypes, in fifty shades of grey. Science. 2013; 339(6119):528-9.

94 Salter SJ, Cox MJ, Turek EM, Calus ST, Cookson WO, Moffatt MF, et al. Reagent and laboratory contamination can critically impact sequence-based microbiome analyses. BMC Biol. 2014 Nov; 12(1):87.

95 Segal LN, Alekseyenko AV, Clemente JC, Kulkarni R, Wu B, Gao Z, et al. Enrichment of lung microbiome with supraglottic taxa is associated with increased pulmonary inflammation. Microbiome. 2013 Jul;1(1):19. 